\title{
Gestational vitamin D deficiency and autism spectrum disorder
}

Anna A. E. Vinkhuyzen, Darryl W. Eyles, Thomas H. J. Burne, Laura M. E. Blanken, Claudia J. Kruithof, Frank Verhulst, Tonya White, Vincent W. Jaddoe, Henning Tiemeier and John J. McGrath

\section{Background}

There is growing interest in linking vitamin D deficiency with autism spectrum disorders (ASDS). The association between vitamin D deficiency during gestation, a critical period in neurodevelopment, and ASD is not well understood.

\section{Aims}

To determine the association between gestational vitamin D status and ASD.

\section{Method}

Based on a birth cohort ( $n=4334)$, we examined the association between 25-hydroxyvitamin D (25OHD), assessed from both maternal mid-gestation sera and neonatal sera, and ASD (defined by clinical records; $n=68$ cases).

\section{Results}

Individuals in the 250HD-deficient group at mid-gestation had more than twofold increased risk of ASD (odds ratio (OR) $=2.42$,
95\% confidence interval (Cl) 1.09 to 5.07, $P=0.03$ ) compared with the sufficient group. The findings persisted in analyses including children of European ethnicity only.

\section{Conclusions}

Mid-gestational vitamin D deficiency was associated with an increased risk of ASD. Because gestational vitamin D deficiency is readily preventable with safe, inexpensive and readily available supplementation, this risk factor warrants closer scrutiny.

\section{Declaration of interest}

None.

\section{Copyright and usage}

(C) The Royal College of Psychiatrists 2017. This is an open access article distributed under the terms of the Creative commons Non-Commercial, No Derivatives (CC BY-NC-ND) license.
Autism spectrum disorder (ASD) is a heterogeneous group of neurodevelopmental disorder characterised by repetitive or stereotyped behaviours, and deficits in social relationships. In recent years, we have learnt a good deal about the genetics of ASD. ${ }^{1}$ Similarly, our knowledge of the non-genetic risk factors associated with ASD has been refined ${ }^{2}$ - candidate prenatal and early life exposures include infection, obstetric complications, and nutritional and toxin-related exposures. Within the domain of nutritional exposures, there is growing interest in the possible links between gestational vitamin D deficiency and an increased risk of ASD. ${ }^{3-6}$ This research has been inspired by birth cohort studies, which have provided evidence that prenatal vitamin D deficiency (as assessed in maternal sera) is associated with a range of later brain-related outcomes including impaired language development ${ }^{7,8}$ and cognitive development in offspring. ${ }^{9,10}$ Although several studies have reported that vitamin D deficiency or insufficiency is more common in children with ASD compared with controls, ${ }^{11}$ few studies addressed the specific hypothesis that vitamin $\mathrm{D}$ deficiency during gestation, a crucial time point in neurodevelopment, is related to the risk of subsequent ASD. One study compared neonatal vitamin D status in children with ASD $v$. their unaffected siblings ( $n=58$ sibling pairs). This study reported lower 25-hydroxyvitamin D (25OHD) concentrations in neonatal samples from the ASD cases compared with their unaffected siblings. ${ }^{6}$ Recently, a Chinese ASD case-control study ( $n=68$ cases) compared first trimester (11-13 weeks) maternal 25OHD concentrations and reported significantly lower concentrations of 25OHD in the ASD cases compared with their gender- and age-matched controls. ${ }^{12}$

We recently reported an association between developmental vitamin $\mathrm{D}$ deficiency and a measure of social impairment within the autism spectrum, based on a large Dutch birth cohort. ${ }^{13}$ In this study, vitamin D was measured at two occasions: at midgestation and at birth. Mid-gestation samples were collected from maternal sera and neonatal sera collected from cord blood. Autistic traits were based on parental ratings of the Social Responsiveness
Scale (SRS), which quantifies behavioural features related to cognition, social communication and autistic mannerisms. ${ }^{14}$ Compared with individuals who were vitamin D sufficient (25OHD concentrations $>50 \mathrm{nmol} / \mathrm{L})$, those who were deficient $(25 \mathrm{OHD}<25 \mathrm{nmol} / \mathrm{L})$ had significantly higher (more impaired) SRS scores.

We had the opportunity to explore the association between developmental vitamin D status and risk of ASD, based on the same Dutch birth cohort in which we previously reported an association between low mid-gestational and neonatal vitamin $\mathrm{D}$ concentrations and autistic traits. ${ }^{13}$ Within the cohort, we had access to an extensive set of potential confounding variables as well as the children's individual-level genome-wide genotype data. These data allowed us to precisely adjust our analyses for important confounding factors including ethnic background and genetic relatedness within the cohort. We predicted that, compared with individuals with sufficient concentrations of 25OHD, those who were $25 \mathrm{OHD}$ deficient (defined as $<25 \mathrm{nmol} / \mathrm{L}$ ) would be at increased risk of clinical ASD.

\section{Method}

\section{The cohort}

This study was embedded in the Generation R Study, a populationbased prospective cohort from fetal life onward, based in Rotterdam, The Netherlands. The design and cohort of Generation $\mathrm{R}$ is extensively described elsewhere. ${ }^{15,16}$ Briefly, the study is designed to identify environmental and genetic correlates of normal and abnormal health-related outcomes in mothers and their children.

A total of 9778 mothers were enrolled in the study, of whom 8878 (91\%) were enrolled during pregnancy. Delivery dates of the pregnant women were between April 2002 and January 2006. Baseline response was $61 \%$ and follow-up rates in children until the age of 6 years exceeded $80 \%$. Relevant to this study, 
Generation $\mathrm{R}$ is a multi-ethnic cohort, with the Dutch comprising roughly half of the sample. The ethnic background of the children was defined by the parents' country of birth and further classified using definitions from Statistics Netherland. ${ }^{17}$ If both parents were born abroad, the country of birth of the mother was used to define the child's ethnicity. The main non-Dutch ethnic groups were Surinamese, Turkish, Moroccan and Cape Verdean, which together accounted for almost $30 \%$ of the cohort. Written informed consent was obtained from the mothers, and the study was approved by the institutional review board of the Erasmus Medical Centre.

\section{Vitamin D status: 25-hydroxyvitamin D}

Vitamin D status was assessed by measuring 25OHD. ${ }^{18}$ Samples were quantified using isotope dilution liquid chromatographytandem mass spectrometry. The analytical system consisted of a Shimadzu Nexera UPLC coupled to an AbSciex 5500 QTRAP equipped with an atmospheric-pressure chemical ionisation source. Assay accuracy was assessed using certified reference materials purchased from the National Institute of Standards and Technology (NIST SRM 972a Levels 1-4). Samples were analysed at the Queensland Brain Institute in Brisbane, Australia, between July 2013 and August 2014. Further details of the assay methodology and a thorough analysis of the prevalence and sociodemographic correlates of $25 \mathrm{OHD}$ concentrations in the Generation $\mathrm{R}$ cohort have been described elsewhere. ${ }^{19} 25 \mathrm{OHD}$ concentration was defined as the sum of 25-hydroxyvitamin $\mathrm{D}_{2}(25 \mathrm{OHD} 2)$ and 25-hydroxyvitamin $\mathrm{D}_{3}$ (25OHD3) species measured in serum. ${ }^{20}$ Within the total Generation R cohort, 25OHD was measured from prenatal and cord serum in a total of 7935 expecting mothers and their children. The first sample was taken at mid-gestation (mean (s.d.) gestational age 20.6 (1.2) weeks; range 18.1-24.9, $n=7256$ ). The second sample was collected at birth, from neonatal cord blood (mean (s.d.) gestational age 40.0 (1.5) weeks; range 27.6-43.6, $n=5023$ ).

In the current analyses, we examined three stratified levels of $25 \mathrm{OHD}$ concentrations: deficient $(<25 \mathrm{nmol} / \mathrm{L})$, insufficient $(25-49.9 \mathrm{nmol} / \mathrm{L})$ and sufficient $(\geq 50 \mathrm{nmol} / \mathrm{L})$. Although there is some debate about the definition of these strata, ${ }^{21}$ there is no debate that children with $25 \mathrm{OHD}$ concentrations of $<25 \mathrm{nmol} / \mathrm{L}$ have vitamin $\mathrm{D}$ deficiency (as defined by an increased risk of rickets and increased parathyroid hormone). Because the fetus is entirely dependent on the maternal supply of $25 \mathrm{OHD}$, and because of the relatively long half-life of 25OHD (approximately 2 weeks), cord blood assays serve as a proxy measure of late gestational 25OHD status (with deficient cord levels suggesting a $25 \mathrm{OHD}$-deficient environment during late gestation). ${ }^{22,23}$

\section{ASD}

The ascertainment of ASD in a general population cohort is challenging. The Netherlands has no general medical register but general practitioners hold all medical records, including information on treatment and diagnoses by medical specialists (e.g. paediatricians and child psychiatrists). A diagnosis of ASD is generally based on clinical consensus by a specialised multidisciplinary team. The diagnostic workup typically involves an extensive developmental case history obtained from parents as well as teachers, based on repeated observations of the child.

To identify ASD cases, we undertook the following steps:

(a) Children were formally screened with the SRS. The authors of the scale recommend cut-offs for screening for ASD in population-based settings, consistent with short-form SRSweighted scores of 1.078 for boys and 1.000 for girls. ${ }^{14}$

(b) Children who scored in the top $15 \%$ on the Child Behavior Checklist-1.5-5 total score ${ }^{24}$ underwent a more specific screening using the Social Communication Questionnaire (SCQ). Children with scores of 15 or above on the SCQ were considered screen-positive. $^{25}$

(c) The presence of psychiatric diagnoses and treatments in the children were routinely assessed from parents at all contact points between ages 6 and 9 years (centre visits and questionnaires).

We obtained medical records of children who were screenpositive on one or more criteria. To minimise false positives, only screen-positive children for whom a diagnosis could be confirmed by medical specialist records were considered ASD cases in this analyses. To minimise false negatives, we relied on multiple sources of information to select the children for medical record retrieval to ascertain ASD.

Within the cohort of children for whom at least one 25OHD assay was available (i.e. at mid-gestation or time of birth), there were (a) 68 screen-positive children on the SRS, of which 23 children with ASD were confirmed with medical records; (b) an additional 20 children were identified screen-positive through the SCQ, of which 6 children with ASD were confirmed with medical records; and (c) an additional 74 children were considered screen-positive based on maternal report, of which 39 children with ASD were confirmed with medical records. In total, these procedures identified 68 children with ASD, within our total sample of 4334 children. The observed prevalence of ASD in the cohort was $1.6 \%$, which is consistent with the estimated prevalence in high-income countries (1.4\%). ${ }^{26}$ To maximise statistical power, children in the cohort who did not meet the screening criteria or children for whom ASD diagnosis was not confirmed were all treated as controls in the downstream analyses.

\section{Ancestry information and genetic relatedness derived from observed common genetic variants}

Ethnicity is strongly associated with vitamin D status (darker skinned individuals are more prone to vitamin D deficiency and insufficiency). ${ }^{19}$ Genome-wide genotype data for the children allow us to build models that are better able to account for variation in ethnic background (compared with register-based or self-ascribed ethnicity) and for genetic relatedness between cohort members that may inflate an association statistic when genes affect the exposure or outcome variable. DNA was extracted from blood collected from the umbilical cord, or if this was not available, from a blood sample that was obtained by venepuncture during the child's visit to the research centre at a mean age of 6 years. Genotyping was performed using Illumina HumanHap 610 or 660 Quad chips, depending on collection time, following manufacturer protocols. A detailed description of the genotyping and quality control of the Generation $\mathrm{R}$ cohort is described elsewhere. ${ }^{27}$ Analyses involving genome-wide genotype data in this study were based on 3234 individuals on 518245 single-nucleotide polymorphisms (SNPs).

\section{Imputation of missing data}

The sample sizes vary between analyses depending on the availability of mid-gestational and cord 25OHD concentrations, offspring genotype information and inclusion criteria related to ethnic background of the children. Note that twin pregnancies and mothers with no follow-up after birth were removed before analyses (see Supplementary Figure 1).

To optimise the sample size (number of ASD cases in our population-based cohort study is small, as expected for a disorder with a low life-time prevalence), we imputed a range of parental and offspring variables that were included as covariates in the models. Imputation was performed using the 'Mix' imputation 
package in $\mathrm{R}^{28}$ 'Mix' uses an iterative multiple regression algorithm which is expectation-maximisation (EM) based and imputes mixed continuous and categorical data under the general location model. Imputation expectancies were based on covariates only and were independent of predictor variables (25OHD concentrations) and outcome variable (ASD status). Four out of eight variables had missing data with missing proportions ranging from 3\% ('Educational level of the mother') to $10 \%$ ('Mother smoking during pregnancy'). We held a conservative approach to imputation of missing variables, as such, we did not impute ethnic background of the child and neither did we impute exposure and outcome variables in the study.

\section{Statistical analyses}

To estimate the effect of vitamin D deficiency on ASD, variations of linear models were fitted to the data. The choice of model depended on the distribution of close relatives in the sample and the method that was applied to control for population structure.

\section{Main analyses}

Logistic regression analyses were applied to test the effect of vitamin D deficiency status on ASD. To remove the effect of family relatedness in the sample, we removed one of each pair of siblings, prioritising siblings with valid vitamin $\mathrm{D}$ measurements from both mid-gestation and cord serum to maximise sample size. Because maximum likelihood estimates are often prone to small sample bias, we applied the Firth correction to our ASD analyses using the 'logistf' package in $\mathrm{R}^{29}$ The Firth correction uses a penalised likelihood estimation method to handle complete or quasi-complete separation in the data.

We compared full models in which we included the vitamin D predictor and parental and offspring demographic variables with nested models that did not include the vitamin $\mathrm{D}$ predictor. Parental variables included maternal and paternal age, maternal body mass index (BMI, measured mid-gestation), smoking status of the mother during pregnancy and educational level of the mother. Offspring variables included ethnicity of the child, gestational age at birth, birth weight and gender of the child. Significance of the vitamin D predictor was tested by comparing the fit of the full model with the fit of the reduced model, thereby considering that the penalty that is involved in the Firth correction is the same for the full and the nested model to make the two models directly comparable.

We fitted four full and reduced (nested) models to test the effect of deficient $v$. sufficient and insufficient $v$. sufficient at mid-gestation or cord. Subsequently, we estimated the population attributable fraction associated with the mid-gestation 25OHD status (assuming that the entire population could be moved into the 25OHD sufficient group), using Equation 10 of Bruzzi and colleagues. ${ }^{30}$

$P A R=1-\frac{1}{x} \sum_{i=1}^{x} \frac{1}{\tilde{r}_{i}}$, with $\mathrm{x}$ being the number of cases and $\tilde{r}_{i}$ being the relative risk for each of the cases in each stratum conditional on covariates.

\section{Sensitivity analyses}

In addition to the main analyses, we undertook three series of sensitivity analyses.

First, we analysed only offspring with European ethnic background (based on the reported parental country of birth). The models fitted to these data were the same as the models in the main analyses, with the exception that the self-report ethnicity variable was not included as a covariate.

Second, because the exposure variable in this study (25OHD concentration) is highly associated with skin colour and thus with ethnic background, ${ }^{19}$ we aimed to fit a model in which potential confounding effects related to ethnic differences between individuals were accounted for accurately. To this end, we fitted a mixed linear model in which we replaced the ethnicity covariate with a genetic component (i.e. genome-wide genetic relationship matrix) that very precisely captures both population stratification and genetic relatedness and consequently allows a test for association that is free from confounding because of sample structure. A detailed description including a simplified worked example of this method is described in our previous work. ${ }^{13}$ Only children for whom individual-level genome-wide genotype data were available were included in these analyses.

Third, we fitted a model on the full sample replacing the categorical vitamin $\mathrm{D}$ predictor with a continuous measure of vitamin $\mathrm{D}$ and tested the association between 25OHD and ASD. These models included all the covariates that were also included in the main analyses.

\section{Results}

Supplementary Figure 1 depicts a flow chart of the inclusion of participants in this study. In total, 4334 children and their mothers were available with measures of vitamin $\mathrm{D}$ concentrations drawn from maternal blood at mid-gestation or drawn from cord blood at time of birth as well as data on the SRS and ASD status. Of these children, 3234 had individual-level genotype data available. Supplementary Table 1 provides an overview of the distributions of parental and offspring demographic variables in the study sample before and after imputation of missing covariates.

On average, 25OHD concentrations from cord blood were lower compared with concentrations at mid-gestation (mean 25OHD concentration in maternal serum at mid-gestation: $58.6 \mathrm{nmol} / \mathrm{L}$; mean 25OHD concentration in cord blood: $35.9 \mathrm{nmol} / \mathrm{L} ; t=35.8$, $P<001)$. The observed correlation between mid-gestation and cord samples was $0.50(t=28.7, P<001)$.

Table 1 summarises the prevalence of $25 \mathrm{OHD}$ deficiency in mid-gestation and cord blood stratified by ASD diagnosis. The proportions of cases and controls with developmental 25OHD deficiency were substantial for both mid-gestation samples and cord samples with higher proportions for ASD cases of midgestation samples but not in cord samples.

\section{Vitamin D and ASD}

For the main analysis, we observed that compared with individuals with sufficient concentrations of $25 \mathrm{OHD}$ at mid-gestation, those that were deficient had a more than twofold increased risk of ASD (odds ratio $(\mathrm{OR})=2.42 ; 95 \%$ confidence interval (CI) 1.09-5.07; $P=0.03$ ) (Table 2). None of the other comparisons were significant.

\begin{tabular}{|c|c|c|}
\hline & $\begin{array}{l}\text { ASD cases } \\
\text { (proportion) }\end{array}$ & $\begin{array}{l}\text { Non-ASD } \\
\text { controls (proportion) }\end{array}$ \\
\hline \multicolumn{3}{|l|}{ Mid-gestation } \\
\hline Deficient & $15(0.24)$ & $639(0.16)$ \\
\hline Insufficient & $13(0.21)$ & $1011(0.26)$ \\
\hline Sufficient & $34(0.55)$ & $2245(0.58)$ \\
\hline Total & 62 & 3895 \\
\hline \multicolumn{3}{|l|}{ Cord } \\
\hline Deficient & $14(0.30)$ & $1040(0.36)$ \\
\hline Insufficient & $21(0.46)$ & $1139(0.40)$ \\
\hline Sufficient & $11(0.24)$ & $691(0.24)$ \\
\hline Total & 46 & 2870 \\
\hline \multicolumn{3}{|c|}{$\begin{array}{l}\text { ASD, autism spectrum disorder; } 25 \mathrm{OHD}, 25 \text {-hydroxyvitamin } \mathrm{D} \text {. } \\
\text { a. Deficient is } 25 \mathrm{OHD} \text { concentrations }<25 \mathrm{nmol} / \mathrm{L} \text {, insufficient is } 25 \mathrm{OHD} \text { concentrations } \\
25 \text { to }<50 \mathrm{nmol} / \mathrm{L} \text {; sufficient is } 250 \mathrm{OHD} \text { concentrations } \geq 50 \mathrm{nmol} / \mathrm{L} \text {. }\end{array}$} \\
\hline
\end{tabular}




\begin{tabular}{|c|c|c|c|c|}
\hline & $n$ & OR $(95 \% \mathrm{Cl})$ & $\chi^{2}(1)$ & $P$ \\
\hline Maternal serum, mid-gestation: deficient $v$. sufficient & 2770 & $2.42(1.09-5.07)$ & 4.64 & $0.03^{*}$ \\
\hline Maternal serum, mid-gestation: insufficient $v$. sufficient & 3103 & $0.86(0.43-1.62)$ & 0.21 & 0.64 \\
\hline Cord blood, at birth: deficient $v$. sufficient & 1673 & $0.94(0.36-2.42)$ & 0.02 & 0.90 \\
\hline Cord blood, at birth: insufficient $v$. sufficient & 1761 & $1.25(0.61-2.69)$ & 0.37 & 0.54 \\
\hline \multicolumn{5}{|c|}{$\begin{array}{l}\text { *Significant at alpha of } 0.05 \text {. } \\
250 \mathrm{HD}, 25 \text {-hydroxyvitamin D. } \\
\text { a. Estimates are based on general linear model; a Firth correction was applied to the model. } \\
\text { b. Covariates included in the model are ethnicity of child, gender of child, birth weight of child, gestational age at time of birth, age of mother at intake, age of father at intake, } \\
\text { smoking history of mother during pregnancy, educational level of mother and body mass index of mother at mid-gestation. } \\
\text { c. Deficient is } 250 \mathrm{HD} \text { concentrations }<25 \mathrm{nmol} / \mathrm{L} \text {, insufficient is } 25 \mathrm{OHD} \text { concentrations from } 25 \text { to }<50 \mathrm{nmol} / \mathrm{L} \text {, sufficient is } 25 \mathrm{OHD} \text { concentrations } \geq 50 \mathrm{nmol} / \mathrm{L} \text {. }\end{array}$} \\
\hline
\end{tabular}

Based on the main analysis, the population attributable risk for the comparisons related to mid-gestation $25 \mathrm{OHD}$ was $10.8 \%$.

\section{Sensitivity analyses}

In the analyses restricted to offspring with European ethnicity, we observed that the pattern of findings persisted. Comparing individuals with deficient levels at mid-gestation with those with sufficient levels, we estimated an OR of 2.42 (95\% CI 1.21-6.43; $P=0.02$ ) (Supplementary Table 2). Similarly, when we replaced ethnicity with the genetic component that captures both population stratification and family relatedness, the pattern of findings identified in the main analyses remained significant, assuring that the observed association is free from confounding with ethnicity. OR for the mid-gestation deficient $v$. sufficient comparison was 2.19 (95\% CI 1.42-3.38; $P=0.01$ ) (Supplementary Table 3). When we treated 25OHD concentrations as a continuous variable, the association between $25 \mathrm{OHD}$ and ASD status was no longer statistically significant $(\mathrm{OR}=0.99,95 \%$ CI $0.98-1.00, \quad P=0.11)$ (Supplementary Table 4).

\section{Discussion}

Based on a sample of 4334 children and their mothers, of whom 68 children were diagnosed with ASD, we show that gestational $25 \mathrm{OHD}$ deficiency is associated with a higher risk of being diagnosed with ASD. This finding is restricted to mid-gestation 25OHD status - in our sample we do not find evidence that cord blood 25OHD status is associated with ASD. In light of the robust findings linking developmental 25OHD concentrations (i.e. both mid-gestation and neonatal 25OHD concentrations) with autistic traits as measured with the SRS in this same population, ${ }^{13}$ this may reflect a lack of power for the current ASD comparisons based on cord blood samples. The number of cases with ASD in the analysis of cord blood was 46 of whom 30\% were 25OHD deficient at time of birth, compared with $36 \%$ of the non-ASD children. Confidence intervals around the estimates are fairly large implying that substantial effect sizes are required to reach statistical significance.

The association between 25OHD deficiency at mid-gestation and ASD was significant and the pattern of findings persisted when we restricted the analyses to the offspring of European ethnicity, and when we thoroughly accounted for ethnic variation and family relatedness using genetic data. These robust sensitivity analyses strongly reduce the chance that our findings were confounded by ethnic diversity and genetic differences in the sample.

Our results add to earlier findings from two previous studies that reported an association between 25OHD deficiency (obtained from neonatal dried blood spots) and 25OHD deficiency in first trimester maternal sera, with increased risk of ASD.,12 Of interest, a Swedish study examined the risk of ASD in the offspring of women with a lifetime diagnosis of $25 \mathrm{OHD}$ deficiency ( $v$. women without this diagnosis). ${ }^{31}$ Although this study was not able to address the specific timing of this exposure (e.g. during the gestation of the index offspring), a robust association was reported between lifetime 25OHD deficiency and autism associated with intellectual disability.

Our study, based on a large, population-based, multi-ethnic cohort, was well suited to test our hypotheses. The prevalence of vitamin $\mathrm{D}$ deficiency was high in this cohort ( $17 \%$ of the mothers had mid-gestational $25 \mathrm{OHD}$ concentrations $<25 \mathrm{nmol} / \mathrm{L}$ ). Based on the current sample and with the standard caveats associated with the interpretation of population attributable fraction (i.e. assuming that the variables of interest are causally related and that other variables remain unchanged), optimising vitamin $\mathrm{D}$ status of this sample could be associated with a reduction in the prevalence of ASD of approximately $10 \%$.

The finding from this study lends weight to the growing body of epidemiological and animal model-based research linking gestational vitamin D deficiency and altered brain development. ASD is considered a neurodevelopmental disorder; abnormalities at different developmental stages may therefore be part of the pathophysiology. Rodent models based on transient prenatal exposure to vitamin D deficiency have found a range of persistent molecular, neurochemical and behavioural changes of interest to neuropsychiatry. ${ }^{32}$ A number of studies have linked ASD with neuronal migration. ${ }^{33}$ Migrationassociated phenotypes such as changes in neuronal density and volume, aberrant minicolumns and heterotopias have been detected in patients with $\mathrm{ASD} .{ }^{34,35}$ As neuronal migration starts at 6 weeks gestation and usually ends around 24 weeks gestation, the observed association between our mid-gestation samples, which were drawn at $\sim 20$ weeks gestation, and risk of ASD corroborates our previous findings linking vitamin $\mathrm{D}$ deficiency with abnormal brain development. Furthermore, neonatal vitamin D deficiency has been previously associated with an increased risk of schizophrenia. ${ }^{36}$ ASD and schizophrenia are known to share genetic risk variants; ${ }^{37}$ our findings provide additional evidence of the shared risk architecture between these two neurodevelopmental disorders. For example, the active form of vitamin $\mathrm{D}(1,25 \mathrm{OHD})$ is known to affect the function of voltage-gated calcium channels. ${ }^{38}$ Variants in genes coding for subunits of these same calcium channels (e.g. CACNA1C) have been linked to risk of both schizophrenia and ASD. ${ }^{39}$

With respect to study limitations, the sample of offspring with ASD was small, and the diagnoses were not confirmed with gold-standard research criteria. Additionally, we lacked resources to systematically retrieve records of all 4334 cohort members for ASD. However, such misclassifications would bias our study towards the null hypothesis (i.e. no association between the variables of interest). Although we had measures of $25 \mathrm{OHD}$ concentrations at two developmental time points, we lacked information at other stages of gestation and early life. In future studies, it will be of interest to explore the critical window during which low vitamin D may contribute to ASD risk - it is feasible that exposure in the first few years of life may also contribute to adverse brain outcomes. Defining the precise critical window during which developmental vitamin $\mathrm{D}$ deficiency adversely affects brain development at this stage would be premature.

This study has several important strengths. The study was based on a large representative multi-ethnic cohort. We used a 
gold standard assessment of 25OHD concentrations and we had access to genome-wide genotype data of the children that allowed us to adjust for population stratification (e.g. ethnic differences) and genetic relatedness between cohort members.

Clearly, our findings are based on a relatively small sample of ASD cases and require replication in a larger sample. Because of the low prevalence of ASD, case-control samples may be better suited to explore this particular hypothesis. Findings based on observational epidemiology remain susceptible to residual confounding and thus randomised controlled trials would be required to infer causality.

Developmental vitamin D deficiency at mid-gestation was associated with a twofold increased risk of ASD. The association between developmental vitamin D deficiency and ASD may have important implications from a public health perspective. It is feasible that a safe, inexpensive and publically acceptable vitamin D supplementation in at-risk groups may reduce the prevalence of this risk factor. Just as prenatal folate supplementation has reduced the incidence of spina bifida, we speculate that prenatal vitamin D supplementation may reduce the incidence of ASD.

Anna A. E. Vinkhuyzen, PhD, Queensland Brain Institute, The University of Queensland, St Lucia, Australia; Institute for Molecular Bioscience, The University of Queensland, St Lucia, Australia; Darryl W. Eyles, PhD, Queensland Brain Institute, The University of Queensland, St Lucia, Australia; Queensland Centre for Mental Health Research, The Park Centre for Mental Health, Wacol, Australia; Thomas H. J. Burne PhD, Queensland Brain Institute, The University of Queensland, St Lucia, Australia; Queensland Centre for Mental Health Research, The Park Centre for Mental Health, Wacol, Australia; Laura M. E. Blanken, MD, MSC, The Generation R Study Group, Erasmus MC, University Medical Center Rotterdam, Rotterdam, The Netherlands; Department of Child and Adolescent Psychiatry/Psychology, Erasmus MC, University Medical Center Rotterdam, Rotterdam, The Netherlands; Claudia J. Kruithof, MSc, The Generation R Study Group, Erasmus MC, University Medical Center Rotterdam, Rotterdam, The Netherlands; Department of Epidemiology, Erasmus MC, University Medical Center Rotterdam, Rotterdam, The Netherlands; Frank Verhulst, MD, PhD, Department of Child and Adolescent Psychiatry/Psychology, Erasmus MC, University Medical Center Rotterdam, Rotterdam, The Netherlands; Tonya White, MD, PhD, Department of Child and Adolescent Psychiatry/Psychology, Erasmus MC, University Medical Center Rotterdam, Rotterdam, The Netherlands; Vincent W. Jaddoe, MD, PhD, The Generation R Study Group, Erasmus MC, University Medical Center Rotterdam, Rotterdam, The Netherlands; Department of Epidemiology, Erasmus MC University Medical Center Rotterdam, Rotterdam, The Netherlands; Department of Pediatrics, Erasmus Medical Center, Rotterdam, The Netherlands; Henning Tiemeier MD, PhD, Department of Epidemiology, Erasmus MC, University Medical Center Rotterdam, Rotterdam, The Netherlands; Department of Child and Adolescent Psychiatry/Psychology, Erasmus MC, University Medical Center Rotterdam, Rotterdam, The Netherlands; John J. McGrath, MD, PhD, Queensland Brain Institute, The University of Queensland, St Lucia, Australia; Queensland Centre for Mental Health Research, The Park Centre for Mental Health, Wacol, Australia

Correspondence: John J. McGrath, Queensland Brain Institute, The University of Queensland, St Lucia QLD 4072, Australia. Email: j.mcgrath@uq.edu.au

First received 21 Oct 2016, final revision 24 Jan 2017, accepted 12 Mar 2017

\section{Acknowledgements}

We thank the participants and staff of the Generation R study. We also thank the members of the Program of Complex Trait Genomics at the Institute for Molecular Bioscience for their support (a list of members of this centre can be found at http://cnsgenomics.com/index.html).

\section{Funding}

The Generation R Study is made possible by financial support from the Erasmus Medica Centre, Rotterdam, the Erasmus University Rotterdam and the Netherlands Organization for Health Research and Development. V.J. received an additional grant from the Netherlands Organization for Health Research and Development (ZonMW-VIDI 016.136.361) and a Consolidator Grant from the European Research Council (ERC-2014-CoG-648916). The vitamin D assay was supported by the National Health and Medical Research Council (NHMRC APP1062846). Shipping of the samples was supported by FP7 Nutrimenthe (grant agreement 212652). The collection of ASD diagnostic information was supported by a Simons Foundation Autism Research Initiative (SFARI - 307280) and a ZonMw TOP grant number 91211021, both to T.W. J.M. received an NHMRC John Cade Fellowship (APP1056929). The researchers are independent from the funders. The study sponsors had no role in the study design, data analysis, interpretation of data or writing of this report.

\section{References}

1 De Rubeis S, Buxbaum JD. Genetics and genomics of autism spectrum disorder: embracing complexity. Hum Mol Genet 2015; 4: R24-31.

2 Lyall K, Croen L, Daniels J, Fallin MD, Ladd-Acosta C, Lee BK, et al. The changing epidemiology of autism spectrum disorders. Annu Rev Public Health 2016; doi: 10.1146/annurev-publhealth-031816-044318 (Epub ahead of print).

3 Cannell JJ. Autism and vitamin D. Med Hypotheses 2008; 70: 750-9.

4 Deluca GC, Kimball SM, Kolasinski J, Ramagopalan SV, Ebers GC. The role of vitamin D in nervous system health and disease. Neuropathol Appl Neurobiol 2013; 39: 458-84

5 Kocovska E, Fernell E, Billstedt E, Minnis H, Gillberg C. Vitamin D and autism: clinical review. Res Dev Disabil 2012; 33: 1541-50.

6 Fernell E, Bejerot S, Westerlund J, Miniscalco C, Simila H, Eyles D, et al. Autism spectrum disorder and low vitamin D at birth: a sibling control study. Mol Autism 2015; 6: 3.

7 Whitehouse AJ, Holt BJ, Serralha M, Holt PG, Hart PH, Kusel MM. Maternal vitamin D levels and the autism phenotype among offspring. J Autism Dev Disord 2013; 43: $1495-504$.

8 Tylavsky FA, Kocak M, Murphy LE, Graff JC, Palmer FB, Volgyi E, et al. Gestational vitamin $25(\mathrm{OH}) \mathrm{D}$ status as a risk factor for receptive language development: a 24-month, longitudinal, observational study. Nutrients 2015; 7: 9918-30.

9 Keim SA, Bodnar LM, Klebanoff MA. Maternal and cord blood 25(OH)-vitamin D concentrations in relation to child development and behaviour. Paediatr Perinat Epidemiol 2014; 28: 434-44.

10 Morales E, Guxens M, Llop S, Rodriguez-Bernal CL, Tardon A, Riaño I, et al. Circulating 25-hydroxyvitamin D3 in pregnancy and infant neuropsychological development. Pediatrics 2012; 130: e913-20.

11 Wang $T$, Shan $L$, Du L, Feng J, Xu Z, Staal WG, et al. Serum concentration of 25-hydroxyvitamin $D$ in autism spectrum disorder: a systematic review and metaanalysis. Eur Child Adolesc Psychiatry 2015; 25: 341-50.

12 Chen J, Xin K, Wei J, Zhang K, Xiao H. Lower maternal serum 25(OH) D in first trimester associated with higher autism risk in Chinese offspring. J Psychosom Res 2016; 89: 98-101.

13 Vinkhuyzen AAE, Eyles DW, Burne THJ, Blanken LM, Kruithof CJ, Verhulst F, et al. Gestational vitamin D deficiency and autism-related traits: the Generation R Study. Mol Psychiatry 2016; doi: 10.1038/mp.2016.213 (Epub ahead of print).

14 Constantino JN, Davis SA, Todd RD, Schindler MK, Gross MM, Brophy SL, et al. Validation of a brief quantitative measure of autistic traits: comparison of the social responsiveness scale with the autism diagnostic interview-revised. J Autism Dev Disord 2003; 33: 427-33.

15 Jaddoe WW, van Duijn $\mathrm{CM}$, Franco $\mathrm{OH}$, van der Heijden $\mathrm{AJ}$, van lizendoorn $\mathrm{MH}$, de Jongste JC, et al. The Generation R Study: design and cohort update 2012. Eur J Epidemiol 2012; 27: 739-56.

16 Kruithof $\mathrm{CJ}$, Kooijman MN, van Duijn $\mathrm{CM}$, Franco $\mathrm{OH}$, de Jongste JC, Klaver $\mathrm{CC}$, et al. The Generation R Study: Biobank update 2015. Eur J Epidemiol 2014; 29 . 911-27.

17 Statistiek CBvd. Immigrants in The Netherlands 2004. National Academies Press, 2004.

18 Institute of Medicine. Dietary Reference Intakes for Calcium and Vitamin D. National Academies Press, 2010.

19 Vinkhuyzen AA, Eyles DW, Burne TH, Blanken LM, Kruithof $C$, Verhulst F, et al. Prevalence and predictors of vitamin $D$ deficiency based on maternal mid gestation and neonatal cord bloods: The Generation R Study. J Steroid Biochem Mol Biol 2016; 164: 161-7.

20 Eyles D, Anderson C, Ko P, Jones A, Thomas A, Burne T, et al. A sensitive LC/MS/ MS assay of $250 \mathrm{H}$ vitamin D-3 and $250 \mathrm{H}$ vitamin D-2 in dried blood spots. Clin Chim Acta 2009; 403: 145-51.

21 Ross AC, Manson JE, Abrams SA, Aloia JF, Brannon PM, Clinton SK, et al. The 2011 report on dietary reference intakes for calcium and vitamin $D$ from the Institute of Medicine: what clinicians need to know. J Clin Endocrinol Metab 2011; 96: 53-8.

22 Hillman LS, Haddad JG. Human perinatal vitamin D metabolism. I. 25-Hydroxyvitamin D in maternal and cord blood. J Pediatr 1974; 84: 742-9.

23 Salle BL, Delvin EE, Lapillonne A, Bishop NJ, Glorieux FH. Perinatal metabolism of vitamin D. Am J Clin Nutr 2000; 71 (5 Suppl): 1317S-24S.

24 Achenbach TM, Edelbrock C. Manual for the Child Behaviour Checklist and Revised Child Behaviour Profile. Department of Psychiatry, University of Vermont, 1983.

25 Berument SK, Rutter $M$, Lord $C$, Pickles A, Bailey $A$. Autism screening questionnaire: diagnostic validity. Br J Psychiatry 1999; 175: 444-51.

26 Developmental Disabilities Monitoring Network Surveillance Year Principal I, Centers for Disease C, Prevention. Prevalence of autism spectrum disorder 
among children aged 8 years - autism and developmental disabilities monitoring network, 11 sites, United States, 2010. MMWR Surveill Summ 2014; 63: 1-21.

27 Medina-Gomez C, Felix JF, Estrada K, Peters MJ, Herrera L, Kruithof CJ, et al. Challenges in conducting genome-wide association studies in highly admixed multi-ethnic populations: The Generation R Study. Eur J Epidemiol 2015; 30 $317-30$

28 Schafer J. Estimation/Multiple Imputation for Mixed Categorical and Continuous Data. R package version 1.09. 2015. (https://cran.r-project.org/web/packages/mix mix.pdf).

29 Heinze G, Schemper M. A solution to the problem of separation in logistic regression. Stat Med 2002; 21: 2409-19.

30 Bruzzi P, Green SB, Byar DP, Brinton LA, Schairer C. Estimating the population attributable risk for multiple risk factors using case-control data. Am J Epidemio 1985; 122: 904-14.

31 Idring S, Rai D, Dal H, Dalman C, Sturm H, Zander E, et al. Autism spectrum disorders in the Stockholm Youth Cohort: design, prevalence and validity. PLOS One 2012; 7: e41280

32 Eyles DW, Burne THJ, McGrath JJ. Vitamin D, effects on brain development, adult brain function and the links between low levels of vitamin $D$ and neuropsychiatric disease. Front Neuroendocrinol 2013; 34: 47-64.

33 Reiner $\mathrm{O}$, Karzbrun E, Kshirsagar A, Kaibuchi K. Regulation of neuronal migration, an emerging topic in autism spectrum disorders. J Neurochem 2016; 136: 440-56.
34 Dicicco-Bloom E, Lord C, Zwaigenbaum L, Courchesne E, Dager SR, Schmitz C et al. The developmental neurobiology of autism spectrum disorder. J Neurosci 2006; 26: 6897-6906.

35 Chen $\mathrm{YH}$, Liao DL, Lai CH, Chen CH. Genetic analysis of AUTS2 as a susceptibility gene of heroin dependence. Drug Alcohol Depend 2013; 128: 238-42.

36 McGrath JJ, Eyles DW, Pedersen CB, Anderson C, Ko P, Burne TH, et al. Neonatal vitamin D status and risk of schizophrenia: a population-based case-control study. Arch Gen Psychiatry 2010; 67: 889-94.

37 Lee SH, Ripke S, Neale BM, Faraone SV, Purcell SM, Perlis RH, et al. Genetic relationship between five psychiatric disorders estimated from genome-wide SNPS. Nat Genet 2013; 45: 984-94.

38 Latimer CS, Brewer LD, Searcy JL, Chen KC, Popovic J, Kraner SD, et al. Vitamin D prevents cognitive decline and enhances hippocampal synaptic function in aging rats. Proc Natl Acad Sci U S A 2014; 111: E4359-66.

39 Casamassima F, Hay AC, Benedetti A, Lattanzi L, Cassano GB, Perlis RH. L-type calcium channels and psychiatric disorders: a brief review. Am J Med Genet $B$ Neuropsychiatr Genet 2010; 153B: 1373-90. 